DOI: $10.17516 / 1997-1397-2020-13-4-480-491$

УДК 519.21

\title{
Rate of the Almost Sure Convergence of a Generalized Regression Estimate Based on Truncated and Functional Data
}

\author{
Halima Boudada* \\ University Freres Mentouri \\ Constantine 1, Algeria \\ Sara Leulmi ${ }^{\dagger}$ \\ LAMASD Laboratory \\ University Freres Mentouri \\ Constantine 1, Algeria \\ Soumia Kharfouch $\ddagger$ \\ University Salah Boubnider \\ Constantine 3, Algeria
}

Received 06.02.2020, received in revised form 25.04.2020, accepted 26.05.2020

\begin{abstract}
In this paper, a nonparametric estimation of a generalized regression function is proposed. The real response random variable (r.v.) is subject to left-truncation by another r.v. while the covariate takes its values in an infinite dimensional space. Under standard assumptions, the pointwise and the uniform almost sure convergences, of the proposed estimator, are established.
\end{abstract}

Keywords: functional data, truncated data, almost sure convergence, local linear estimator.

Citation: H. Boudada, S. Leulmi, S. Kharfouchi, Rate of the Almost Sure Convergence of a Generalized Regression Estimate Based on Truncated and Functional Data, J. Sib. Fed. Univ. Math. Phys., 2020, 13(4), 480-491. DOI: 10.17516/1997-1397-2020-13-4-480-491.

\section{Introduction and preliminaries}

The investigation of the link between a scalar variable of interest $Y$ and a functional covariate $X$ is among the most famous nonparametric statistical works in the last two decades. We mention [1] who proposed a new version of the estimator of the regression operator $m(x)=E(Y / X=x)$, in the case of independent and identically distributed (i.i.d.) observations, and studied its almost complete convergence. They used the so called local linear method.

In the case of complete data, many works followed this last method. For example, in [9] the uniform almost-complete convergence of the local linear conditional quantile estimator was established, while in [8] the case of a generalized regression function with functional dependent data was considered. The asymptotic normality of the local linear estimator of the conditional density for functional time series data was studied in [12] and both the pointwise and the uniform almost complete convergences, of a generalized regression estimate, were investigated in [7]. All these studies were carried in the case of complete data, however in practice, one or more truncation variables may interfere with the variable of interest and prevent its observation in a complete manner. In this setting of truncation model, one can find many works such as that

\footnotetext{
*boudadahalima@yahoo.fr

${ }^{\dagger}$ math17sara@yahoo.fr

${ }^{\ddagger}$ kharfouchi@yahoo.fr

(C) Siberian Federal University. All rights reserved
} 
of [5] where a kernel conditional quantile estimator was proposed and its strong uniform almost sure convergence established. Similarly, [2] studied the almost complete convergence rate and the asymptotic normality of a family of nonparametric estimators for the $\psi$-regression model. But, as far as we know, the local linear method has not been investigated for truncated data.

Hence, our goal is to propose a generalized regression estimator, when the response variable is subject to left-truncation, and to establish both its pointwise and its uniform almost sure convergences.

To this end, this article is ordered as follows. In Section 2, we recall some basic knowledge of the left -truncation model and we construct our local linear estimator. Section 3 is devoted to prove its pointwise almost sure convergence. Finally, its uniform convergence is established in Section 4.

To make things more easier for readers, we give the definition of the almost complete convergence:

Let $\left(W_{n}\right)_{n \in \mathbb{N} *}$ be a sequence of real random variables r.r.v.. We say that $\left(W_{n}\right)_{n \in \mathbb{N} *}$ converges almost completely to some r.r.v. $W$, and we note $W_{n} \longrightarrow^{a . c o .} W$, if and only if $\forall \epsilon>0$, $\sum_{n=1}^{\infty} P\left(\left|W_{n}-W\right|>\epsilon\right)<\infty$. Moreover, let $\left(v_{n}\right)_{n \in \mathbb{N} *}$ be a sequence of positive real numbers going to zero; we say that the rate of the almost complete convergence of $\left(W_{n}\right)_{n \in \mathbb{N} *}$ to $W$ is of order $\left(v_{n}\right)$ and we note $W_{n}-W=O_{a . c o}\left(v_{n}\right)$, if and only if $\exists \epsilon_{0}>0, \sum_{n=1}^{\infty} P\left(\left|W_{n}-W\right|>\epsilon_{0} v_{n}\right)<\infty$. It is clear, from Borel Cantelli lemma, that this convergence is stronger than the almost-sure one (a.s.).

\section{Estimation}

Let $\left(X_{i}, Y_{i}\right)$ for $i=1, \ldots, N$, be $N$ identical and independent couples distributed as $(X, Y)$ which takes its values in $\mathcal{F} \times \mathbb{R}$, where $\mathcal{F}$ is a semi metric space endowed with a semi metric $d$. The unknown distribution function (d.f.) of $Y$ is denoted by $F$.

Let $T$ be another r.v. which has unknown d.f. $G$ and $\left(T_{i}\right)_{i=1, \ldots, N}$ be a sample of i.i.d. random variables that are distributed as $T$. $T$ is supposed independent of $(X, Y) . N$ is unknown but deterministic. In the left truncation model, the lifetime $Y_{i}$ and the truncation r.v. $T_{i}$ are both observable only when $Y_{i} \geqslant T_{i}$. We denote $\left(Y_{i}, T_{i}\right), i=1,2, \ldots, n(n \leqslant N)$ the actual observed sample of size $n$ which, as a consequence of truncation, is a binomial r.v. with parameters $N$ and $\mu=\mathbb{P}(Y \geqslant T)$. It is clear that if $\mu=0$, no data can be observed, and therefore, we suppose throughout this article that $\mu>0$.

By the strong law of large numbers, we have

$$
\widehat{\mu}_{n}:=\frac{n}{N} \rightarrow \mu, \mathbb{P}-p . s .
$$

We point out that if the original data $\left(Y_{i}, T_{i}\right), i=1,2, \ldots, N$ are i.i.d., the observed data $\left(Y_{i}, T_{i}\right)$, $i=1,2, \ldots, n$ are still i.i.d. (see $[6]$ ).

Under random left truncation model, following [10], the d.f.s of $\mathrm{Y}$ and $\mathrm{T}$ are expressed respectively as,

$$
F^{*}(y)=\mu^{-1} \int_{-\infty}^{y} G(u) d F(u) \quad \text { and } \quad G^{*}(t)=\mu^{-1} \int_{-\infty}^{\infty} G(t \wedge u) d F(u),
$$

where $t \wedge u=\min (t, u)$ and are estimated by their empirical estimators,

$$
F_{n}^{*}(y)=n^{-1} \sum_{i=1}^{n} 1_{\left\{Y_{i} \leqslant y\right\}} \quad \text { and } \quad G_{n}^{*}(t)=n^{-1} \sum_{i=1}^{n} 1_{\left\{T_{i} \leqslant t\right\}} .
$$


Define

$$
C(y):=G^{*}(y)-F^{*}(y)=\mu^{-1} G(y)(1-F(y)),
$$

the empirical estimator of $C(y)$ is defined by

$$
C_{n}(y)=n^{-1} \sum_{i=1}^{n} 1_{\left\{T_{i} \leqslant y \leqslant Y_{i}\right\}}
$$

The nonparametric maximum likelihood estimators of $F$ and $G$ are given respectively by

$$
F_{n}(y)=1-\prod_{i / Y_{i} \leqslant y}\left[\frac{n C_{n}\left(Y_{i}\right)-1}{n C_{n}\left(Y_{i}\right)}\right] \quad \text { and } \quad G_{n}(y)=\prod_{i / T_{i}>y}\left[\frac{n C_{n}\left(T_{i}\right)-1}{n C_{n}\left(T_{i}\right)}\right]
$$

According to [4], $\mu$ can be estimated by

$$
\mu_{n}=C_{n}^{-1}(y) G_{n}(y)\left(1-F_{n}(y)\right)
$$

which is independent of $y$.

Our results will be stated with respect to the conditional probability $\mathbf{P}($.$) related to the n$ sample instead of the probability measure $\mathbb{P}($.$) related to the N$-sample. We donate by $\mathbf{E}$ and $\mathbb{E}$ the respective expectation operators of $\mathbf{P}($.$) and \mathbb{P}($.$) .$

For any d.f. $L$, let $a_{L}=\inf \{y: L(y)>0\}$ and $b_{L}=\sup \{y: L(y)<1\}$ be its two endpoints. The asymptotic properties of $F_{n}, G_{n}$ and $\mu_{n}$ are obtained only if $a_{G} \leqslant a_{F}$ and $b_{G} \leqslant b_{F}$. We take two real numbers $c$ and $d$ such that $[c, d] \subset\left[a_{F}, b_{F}\right]$, we are going to use this inclusion in the uniform consistency of the distribution law $G($.$) of the truncated r.v. T$ which is stated over a compact set (see Remark 6 in [11]).

Hence, based on the idea of the Nadaraya-Watson kernel smoother, the estimator of the general regression function $m_{\varphi}(x)$ defined, for all $x \in \mathcal{F}$, by $m_{\varphi}(x)=E(\varphi(Y) / X=x)$, where $\varphi$ is a known real-valued borel function, is defined by

$$
\widehat{m}_{\varphi}(x)=\frac{\sum_{i=1}^{n} \varphi\left(Y_{i}\right) K\left(h^{-1} d\left(X_{i}, x\right)\right) G_{n}^{-1}\left(Y_{i}\right)}{\sum_{i=1}^{n} K\left(h^{-1} d\left(X_{i}, x\right)\right) G_{n}^{-1}\left(Y_{i}\right)}
$$

where $K$ is a standard univariate kernel function and the bandwidth $h:=h_{n}$ is a sequence of strictly positive real numbers which plays a smoothing parameter role.

Note that all the sums containing $G_{n}^{-1}\left(Y_{i}\right)$ are taken for $i$ such that $G_{n}\left(Y_{i}\right) \neq 0$.

Following [1] and [7], the local linear estimator of $m_{\varphi}$ in the case of truncated data is obtained as the solution for $a$ of the following minimization problem

$$
\min _{(a, b) \in \mathbb{R}^{2}} \sum_{i=1}^{n}\left(\varphi\left(Y_{i}\right)-a-b \beta\left(X_{i}, x\right)\right)^{2} K\left(h^{-1} d\left(X_{i}, x\right)\right) G_{n}^{-1}\left(Y_{i}\right),
$$

where $\beta(.,$.$) is a known operator from \mathcal{F} \times \mathcal{F}$ into $\mathbb{R}$ such that, $\forall x \in \mathcal{F}, \beta(x, x)=0$.

By a simple calculus, one can derive the following explicit estimator

$$
\widehat{m}_{\varphi}(x)=\frac{\sum_{i, j=1}^{n} W_{i j}(x) \varphi\left(Y_{j}\right)}{\sum_{i, j=1}^{n} W_{i j}(x)} \quad\left(\frac{0}{0}:=0\right),
$$

where

$$
W_{i j}(x)=\Delta_{i j}(x) G_{n}^{-1}\left(Y_{i}\right) G_{n}^{-1}\left(Y_{j}\right)
$$

with

$$
\Delta_{i j}(x):=\beta\left(X_{i}, x\right)\left(\beta\left(X_{i}, x\right)-\beta\left(X_{j}, x\right)\right) K\left(h^{-1} d\left(X_{i}, x\right)\right) K\left(h^{-1} d\left(X_{j}, x\right)\right)
$$




\section{Pointwise almost sure corvengence}

For any positive real $h$, let $B(x, h):=\{y \in \mathcal{F} / d(x, y) \leqslant h\}$ be a closed ball in $\mathcal{F}$ of center $x$ and radius $h, \Phi_{x}\left(h, h^{\prime}\right):=P\left(h \leqslant d(x, X) \leqslant h^{\prime}\right)$ and $\Phi_{x}(h):=\Phi_{x}(0, h)$.

To establish the asymptotic behaviour of our estimator $\widehat{m}_{\varphi}(x)$ for a fixed point $x$ in $\mathcal{F}$, we use the following assumptions:

(H1) For any $h>0 ; \Phi_{x}(h)>0$.

(H2) There exists $b>0$ such that for all $x_{1}, x_{2} \in B(x, h) ;\left|m_{\varphi}\left(x_{1}\right)-m_{\varphi}\left(x_{2}\right)\right| \leqslant C_{x} d^{b}\left(x_{1}, x_{2}\right)$ where $C_{x}$ is a positive constant depending on $x$.

(H3) The function $\beta(.,$.$) is such that$

$$
\exists 0<M_{1}<M_{2}, \forall x^{\prime} \in \mathcal{F}, M_{1} d\left(x, x^{\prime}\right) \leqslant\left|\beta\left(x, x^{\prime}\right)\right| \leqslant M_{2} d\left(x, x^{\prime}\right) .
$$

(H4) The kernel $K$ is a positive and differentiable function on its support $[0,1]$.

(H5) The bandwidth $h$ satisfies $\lim _{n \rightarrow \infty} h=0$ and $\lim _{n \rightarrow \infty}\left(\sqrt{\frac{\ln n}{n \Phi_{x}(h)}}\right)=0$.

(H6) There exists an integer $n_{0}$, such that

$$
\forall n>n_{0}, \frac{1}{\Phi_{x}(h)} \int_{0}^{1} \Phi_{x}(z h, h) \frac{d}{d z}\left(z^{2} K(z)\right)>0 .
$$

(H7) $h \int_{B(x, h)} \beta(u, x) d \mathbf{P}_{X}(u)=o\left(\int_{B(x, h)} \beta^{2}(u, x) d \mathbf{P}_{X}(u)\right)$, where $d \mathbf{P}_{X}$ is the distribution of $X$.

(H8) $\forall m \geqslant 2 ; \sigma_{m}: x \longmapsto \mathbf{E}\left(\left|\varphi^{m}(Y)\right| / X\right)$ is a continuous operator on $\mathcal{F}$.

Remark 3.1. Hypotheses (H1)-(H5) are standard in the nonparametric functional regression setting. The rest of the hypotheses have already been used in the literature, we refer for (H6) and (H7) to [1] and for (H8) to [7].

Theorem 3.1. Assume that assumptions (H1)-(H8) are satisfied, then

$$
\widehat{m}_{\varphi(x)}-m_{\varphi(x)}=O\left(h^{b}\right)+O_{a . s .}\left(\sqrt{\frac{\ln n}{n \Phi_{x}(h)}}\right) .
$$

We remark that to prove our theorem we need to define the following pseudo-estimators

$$
r_{l}(x)=\frac{\mu_{n}^{2}}{n(n-1) \mathbf{E}\left(\Delta_{12}(x)\right)} \sum_{i \neq j} G_{n}^{-1}\left(Y_{i}\right) G_{n}^{-1}\left(Y_{j}\right) \Delta_{i j}(x) \varphi^{l}\left(Y_{j}\right)
$$

and

$$
\tilde{m}_{l}(x)=\frac{\mu^{2}}{n(n-1) \mathbf{E}\left(\Delta_{12}(x)\right)} \sum_{i \neq j} G^{-1}\left(Y_{i}\right) G^{-1}\left(Y_{j}\right) \Delta_{i j}(x) \varphi^{l}\left(Y_{j}\right), \quad \text { for } \quad l=0,1 .
$$

Consider the following decomposition

$$
\begin{aligned}
& \widehat{m}_{\varphi}(x)-m_{\varphi}(x)=\frac{r_{1}(x)}{r_{0}(x)}-m_{\varphi}(x)= \\
&=\frac{1}{r_{0}(x)}\left\{r_{1}(x)-\tilde{m}_{1}(x)\right\}+\frac{1}{r_{0}(x)}\left\{\tilde{m}_{1}(x)-\mathbf{E}\left(\tilde{m}_{1}(x)\right)\right\}+\frac{1}{r_{0}(x)}\left\{\mathbf{E}\left(\tilde{m}_{1}(x)\right)-m_{\varphi}(x)\right\}+ \\
& \quad+\frac{m_{\varphi}(x)}{r_{0}(x)}\left\{\left(\tilde{m}_{0}(x)-r_{0}(x)\right)+\left(\mathbf{E}\left(\tilde{m}_{0}(x)\right)-\tilde{m}_{0}(x)\right)+\left(-\mathbf{E}\left(\tilde{m}_{0}(x)\right)+1\right)\right\} .
\end{aligned}
$$


Moreover, we note for any $x \in \mathcal{F}$ and for all $i=1, \ldots, n$

$$
K_{i}(x):=K\left(h^{-1} d\left(X_{i}, x\right)\right) \quad \text { and } \quad \beta_{i}(x):=\beta\left(X_{i}, x\right) .
$$

To make things easier, we introduce the following lemmas.

Lemma 1. Under the assumptions (H1)-(H8), we have

$$
\left|r_{l}(x)-\tilde{m}_{l}(x)\right|=O_{a . s .}\left(\sqrt{\frac{\ln n}{n \Phi_{x}(h)}}\right) .
$$

Proof. For $l=0,1$

$$
\begin{aligned}
\left|r_{l}(x)-\tilde{m}_{l}(x)\right| & =\mid \frac{\mu_{n}^{2}}{n(n-1) \mathbf{E}\left(\Delta_{12}(x)\right)} \sum_{i \neq j} G_{n}^{-1}\left(Y_{i}\right) G_{n}^{-1}\left(Y_{j}\right) \Delta_{i j}(x) \varphi^{l}\left(Y_{j}\right)- \\
& -\frac{\mu^{2}}{n(n-1) \mathbf{E}\left(\Delta_{12}(x)\right)} \sum_{i \neq j} G^{-1}\left(Y_{i}\right) G^{-1}\left(Y_{j}\right) \Delta_{i j}(x) \varphi^{l}\left(Y_{j}\right) \mid \leqslant \\
& \leqslant\left[\frac{\left|\mu_{n}^{2}-\mu^{2}\right|}{G_{n}^{2}\left(a_{F}\right)}+\mu^{2}\left(\frac{\sup _{y \in[c, d]}\left|G_{n}^{2}(y)-G^{2}(y)\right|}{G^{2}\left(a_{F}\right) G_{n}^{2}\left(a_{F}\right)}\right)\right] \times \\
& \times \sum_{i \neq j}\left|\frac{\Delta_{i j}(x) \varphi^{l}\left(Y_{j}\right)}{n(n-1) \mathbf{E}\left(\Delta_{12}(x)\right)}\right| .
\end{aligned}
$$

From Theorem 3.2 of [4] we have $\left|\mu_{n}-\mu\right|=O_{a . s}\left(n^{-1 / 2}\right)$, while Remark 6 of [11] gives $\left|G_{n}\left(a_{F}\right)-G\left(a_{F}\right)\right|=O_{a . s}\left(n^{-1 / 2}\right)$ which are negligible with respect to $O\left(\sqrt{\frac{\ln n}{n \Phi_{x}(h)}}\right)$. The rest of the proof is completed in [7]. Thus, we have $\left|r_{l}(x)-\tilde{m}_{l}(x)\right|=O_{a . s .}\left(\sqrt{\frac{\ln n}{n \Phi_{x}(h)}}\right)$.

Lemma 2. Under the assupmtions (H1), (H2) and (H4), we obtain

$$
\left|\boldsymbol{E}\left(\tilde{m}_{1}(x)\right)-m_{\varphi}(x)\right|=O\left(h^{b}\right) .
$$

Proof. We have

$$
\begin{aligned}
\mathbf{E}\left(\tilde{m}_{1}(x)\right) & =\mathbf{E}\left(\frac{\mu^{2}}{n(n-1) \mathbf{E}\left(\Delta_{12}(x)\right)} \sum_{i \neq j} G^{-1}\left(Y_{i}\right) G^{-1}\left(Y_{j}\right) \Delta_{i j}(x) \varphi\left(Y_{j}\right)\right)= \\
& =\frac{\mu^{2}}{\mathbf{E}\left(\Delta_{12}(x)\right)} \mathbf{E}\left(\frac{1}{G\left(Y_{1}\right) G\left(Y_{2}\right)} \Delta_{12}(x) \varphi\left(Y_{2}\right)\right)= \\
& =\frac{\mu^{2}}{\mathbf{E}\left(\Delta_{12}(x)\right)} \mathbf{E}\left[\mathbb{E}\left(\Delta_{12}(x) \varphi\left(Y_{2}\right) \frac{1_{\left\{Y_{1} \geqslant T_{1}\right\}} 1_{\left\{Y_{2} \geqslant T_{2}\right\}}}{\mu^{2} G\left(Y_{1}\right) G\left(Y_{2}\right)} / \sigma\left(X_{1}, Y_{1}, X_{2}, Y_{2}\right)\right)\right]= \\
& =\frac{1}{\mathbf{E}\left(\Delta_{12}(x)\right)} \mathbf{E}\left(\Delta_{12}(x) m_{\varphi}\left(X_{2}\right)\right) .
\end{aligned}
$$

So we can write, under assumption (H4)

$$
\begin{aligned}
\left|m_{\varphi}(x)-\mathbf{E}\left(\tilde{m}_{1}(x)\right)\right| & =\frac{1}{\left|\mathbf{E}\left(\Delta_{12}(x)\right)\right|}\left|\mathbf{E}\left(\Delta_{12}(x)\left(m_{\varphi}(x)-m_{\varphi}\left(X_{2}\right)\right)\right)\right| \leqslant \\
& \leqslant \sup _{x^{\prime} \in B(x, h)}\left|m_{\varphi}(x)-m_{\varphi}\left(x^{\prime}\right)\right| .
\end{aligned}
$$

Using (H2), we obtain $\left|\mathbf{E}\left(\tilde{m}_{1}(x)\right)-m_{\varphi}(x)\right|=O\left(h^{b}\right)$. 
Lemma 3. i) Under the assumptions (H1)-(H8), we get

$$
\tilde{m}_{1}(x)-\boldsymbol{E}\left(\tilde{m}_{1}(x)\right)=O_{a . c o}\left(\sqrt{\frac{\ln n}{n \Phi_{x}(h)}}\right) .
$$

ii) Under the assumptions (H1), (H3)-(H7), we obtain

$$
\begin{gathered}
\tilde{m}_{0}(x)-1=O_{a . c o}\left(\sqrt{\frac{\ln n}{n \Phi_{x}(h)}}\right) \quad \text { and } \\
\exists \vartheta>0, \text { suchthat } \sum_{n=1}^{\infty} \boldsymbol{P}\left(\tilde{m}_{0}(x)<\vartheta\right)<\infty .
\end{gathered}
$$

Proof. Remark that

$$
\tilde{m}_{1}(x)=Q(x)\left[M_{2,1}(x) M_{4,0}(x)-M_{3,1}(x) M_{3,0}(x)\right],
$$

where for $p=2,3,4$ and $l=0,1$

$$
Q(x)=\frac{n^{2} h^{2} \Phi_{x}^{2}(h)}{n(n-1) \mathbf{E}\left(\Delta_{12}(x)\right)}
$$

and

$$
M_{p, l}(x)=\frac{1}{n \Phi_{x}(h)} \sum_{i=1}^{n} \frac{\mu K_{i}(x) \beta_{i}^{p-2}(x) \varphi^{l}\left(Y_{i}\right)}{h^{p-2} G\left(Y_{i}\right)} .
$$

So, we have

$$
\begin{aligned}
\tilde{m}_{1}(x)-\mathbf{E}\left(\tilde{m}_{1}(x)\right) & =Q(x)\left\{M_{2,1}(x) M_{4,0}(x)-\mathbf{E}\left(M_{2,1}(x) M_{4,0}(x)\right)\right\}- \\
& -Q(x)\left\{M_{3,1}(x) M_{3,0}(x)-\mathbf{E}\left(M_{3,1}(x) M_{3,0}(x)\right)\right\} .
\end{aligned}
$$

Notice that $Q(x)=O(1)$, see the proof of Lemma (4.4) of [1].

We need to prove that for $p=2,3,4$ and $l=0,1$

$$
\begin{aligned}
& \mathbf{E}\left(M_{p, l}(x)\right)=O(1) ; M_{p, l}(x)-\mathbf{E}\left(M_{p, l}(x)\right)=O_{a . c o}\left(\sqrt{\frac{\ln n}{n \Phi_{x}(h)}}\right), \\
& \mathbf{E}\left(M_{2,1}(x)\right) \mathbf{E}\left(M_{4,0}(x)\right)-\mathbf{E}\left(M_{2,1}(x) M_{4,0}(x)\right)=O\left(\sqrt{\frac{\ln n}{n \Phi_{x}(h)}}\right), \\
& \mathbf{E}\left(M_{3,1}(x)\right) \mathbf{E}\left(M_{3,0}(x)\right)-\mathbf{E}\left(M_{3,1}(x) M_{3,0}(x)\right)=O\left(\sqrt{\frac{\ln n}{n \Phi_{x}(h)}}\right) .
\end{aligned}
$$

- Using assumptions (H1)-(H4), we can easily have for $p=2,3,4$ and $l=0,1$

$$
\begin{aligned}
\mathbf{E}\left(M_{p, l}(x)\right) & =\mathbf{E}\left(\frac{1}{\Phi_{x}(h)} \sum_{i=1}^{n} \frac{\mu K_{i}(x) \beta_{i}^{p-2}(x) \varphi^{l}\left(Y_{i}\right)}{h^{p-2} G\left(Y_{i}\right)}\right)= \\
& =\mu h^{2-p} \Phi_{x}^{-1}(h) \mathbf{E}\left[\mathbb{E}\left(K_{1}(x) \beta_{1}^{p-2}(x) \varphi^{l}\left(Y_{1}\right) \frac{1_{\left\{Y_{1} \geqslant T_{1}\right\}}}{\mu G\left(Y_{1}\right)} / \sigma\left(X_{1}, Y_{1}\right)\right)\right]= \\
& =h^{2-p} \Phi_{x}^{-1}(h) \mathbf{E}\left(K_{1}(x) \beta_{1}^{p-2}(x) m_{\varphi}^{l}\left(X_{1}\right)\right) .
\end{aligned}
$$

Lemma A.1 (i) in [1] and the condition (H2) allow us to get $\mathbf{E}\left(M_{p, l}(x)\right)=O(1)$. 
- Treatment of the term $M_{p, l}(x)-\mathbf{E}\left(M_{p, l}(x)\right)$. We put

$$
M_{p, l}(x)-\mathbf{E}\left(M_{p, l}(x)\right)=\frac{1}{n} \sum_{i=1}^{n} Z_{i}^{(p, l)}(x),
$$

where

$$
Z_{i}^{(p, l)}(x)=\frac{1}{h^{p-2} \Phi_{x}(h)}\left\{\frac{\mu K_{i}(x) \beta_{i}^{p-2}(x) \varphi^{l}\left(Y_{i}\right)}{G\left(Y_{i}\right)}-\mathbf{E}\left(\frac{\mu K_{i}(x) \beta_{i}^{p-2}(x) \varphi^{l}\left(Y_{i}\right)}{G\left(Y_{i}\right)}\right)\right\} .
$$

The main point is to evaluate asymptotically the mth-order moment of the r.r.v. $Z_{i}^{(p, l)}(x)$. By using Lemma A.1 (i) in [1], we have

$$
\begin{aligned}
\mathbf{E}\left|\left\{Z_{i}^{(p, l)}(x)\right\}^{m}\right|= & h^{(-p+2) m} \Phi_{x}^{-m}(h) \mathbf{E} \mid \sum_{k=0}^{m} C_{m}^{k}(-1)^{m-k}\left(\frac{\mu K_{i}(x) \beta_{i}^{p-2}(x) \varphi^{l}\left(Y_{i}\right)}{G\left(Y_{i}\right)}\right)^{k} \times \\
& \times\left(\mathbf{E}\left[\frac{\mu K_{i}(x) \beta_{i}^{p-2}(x) \varphi^{l}\left(Y_{i}\right)}{G\left(Y_{i}\right)}\right]\right)^{m-k} \mid= \\
= & O\left(\Phi_{x}^{(-m+1)}(h)\right) .
\end{aligned}
$$

Finally, it suffices to apply Corollary A.8 (ii) in [3] with $a_{n}^{2}=\Phi_{x}^{(-1)}(h)$ to get, for $p \in\{2,3,4\}$ and $l \in\{0,1\}$

$$
M_{p, l}(x)-\mathbf{E}\left(M_{p, l}(x)\right)=O_{a . c o}\left(\sqrt{\frac{\ln n}{n \Phi_{x}(h)}}\right) .
$$

- Moving to study the term $\mathbf{E}\left(M_{2,1}(x)\right) \mathbf{E}\left(M_{4,0}(x)\right)-\mathbf{E}\left(M_{2,1}(x) M_{4,0}(x)\right)$, we have

$$
\begin{aligned}
& \mathbf{E}\left(M_{2,1}(x)\right) \mathbf{E}\left(M_{4,0}(x)\right)-\mathbf{E}\left(M_{2,1}(x) M_{4,0}(x)\right)= \\
& =n^{-1} h^{-2} \Phi_{x}^{-2}(h) \mathbf{E}\left(K_{1}(x) \beta_{1}^{2}(x)\right) \mathbf{E}\left(K_{1}(x) \varphi\left(Y_{1}\right)\right)+O\left(\left(n \Phi_{x}(h)\right)^{-1}\right),
\end{aligned}
$$

by using similar arguments as previously, we get

$$
\mathbf{E}\left(M_{2,1}(x)\right) \mathbf{E}\left(M_{4,0}(x)\right)-\mathbf{E}\left(M_{2,1}(x) M_{4,0}(x)\right)=O\left(\left(n \Phi_{x}(h)\right)^{-1}\right),
$$

which is, under $(\mathrm{H} 5)$, negligible with respect to $O\left(\sqrt{\frac{\ln n}{n \Phi_{x}(h)}}\right)$.

- By similar arguments, one can prove that

$$
\mathbf{E}\left(M_{3,1}(x)\right) \mathbf{E}\left(M_{3,0}(x)\right)-\mathbf{E}\left(M_{3,1}(x) M_{3,0}(x)\right)=O\left(\sqrt{\frac{\ln n}{n \Phi_{x}(h)}}\right) .
$$

For the second part of the lemma, it's easy to find that $\mathbf{E}\left(\tilde{m}_{0}(x)\right)=1$ and this leads us to get the last result.

Theorem 3.1 is proved. 


\section{Uniform almost sure convergence}

In this section, we will investigate the uniform almost sure convergence of $\widehat{m}_{\varphi}$ on some subset $\mathcal{S}_{\mathcal{F}}$ of $\mathcal{F}$, such that $\mathcal{S}_{\mathcal{F}} \subset \bigcup_{k=1}^{d_{n}} B\left(x_{k}, r_{n}\right)$, where $x_{k} \in \mathcal{S}_{\mathcal{F}}$ and $r_{n}$ (respectively $d_{n}$ ) is a sequence of positive real (respectively integer) numbers. For this, we need the following assumptions.

(U1) There exist a differentiable function $\Phi$ and strictly positive constants $C, C_{1}$ and $C_{2}$ such that

$$
\forall x \in \mathcal{S}_{\mathcal{F}}, \forall h>0 ; \quad 0<C_{1} \Phi(h) \leqslant \Phi_{x}(h) \leqslant C_{2} \Phi(h)<\infty
$$

and

$$
\exists \eta_{0}>0, \forall \eta<\eta_{0}, \Phi^{\prime}(\eta)<C,
$$

where $\Phi^{\prime}$ denotes the first derivative of $\Phi$ with $\Phi(0)=0$.

(U2) The generalized regression function $m_{\varphi}$ satisfies

$$
\exists C>0, \exists b>0, \forall x \in \mathcal{S}_{\mathcal{F}}, x^{\prime} \in B(x, h),\left|m_{\varphi}(x)-m_{\varphi}\left(x^{\prime}\right)\right| \leqslant C d^{b}\left(x, x^{\prime}\right) .
$$

(U3) The function $\beta(.,$.$) satisfies (H3) uniformly on x$ and the following Lipschitz's condition

$$
\exists C>0, \forall x_{1} \in \mathcal{S}_{\mathcal{F}}, x_{2} \in \mathcal{S}_{\mathcal{F}}, x \in \mathcal{F},\left|\beta\left(x, x_{1}\right)-\beta\left(x, x_{2}\right)\right| \leqslant C d\left(x_{1}, x_{2}\right) .
$$

(U4) The kernel $K$ fulfils (H4) and is Lipschitzian on $[0,1]$.

(U5) $\lim _{n \longrightarrow \infty} h=0$, and for $r_{n}=O\left(\frac{\ln n}{n}\right)$, we have for $n$ large enough

$$
\frac{(\ln n)^{2}}{n \Phi(h)}<\ln d_{n}<\frac{n \Phi(h)}{\ln n}
$$

and

$$
\sum_{n=1}^{\infty} d_{n}^{(1-\beta)}<\infty \text { for some } \beta>1 .
$$

(U6) The bandwidth $h$ satisfies $\exists n_{0} \in \mathbb{N}, \exists C>0$, such that

$$
\forall n>n_{0}, \forall x \in \mathcal{S}_{\mathcal{F}}, \frac{1}{\Phi_{x}(h)} \int_{0}^{1} \Phi_{x}(z h, h) \frac{d}{d z}\left(z^{2} K(z)\right)>C>0
$$

and

$$
h \int_{B(x, h)} \beta(u, x) d \mathbf{P}_{X}(u)=o\left(\int_{B(x, h)} \beta^{2}(u, x) d \mathbf{P}_{X}(u)\right)
$$

uniformly on $x$.

(U7) $\exists C>0$ such that $\forall m \geqslant 2: \mathbf{E}\left(\left|\varphi^{m}(Y)\right| / X=x\right)<v_{m}(x)<C<\infty$ with $v_{m}($.$) continuous$ on $\mathcal{S}_{\mathcal{F}}$.

Remark 4.1. These hypothesis are the uniform version of the assumed conditions in the pointwise case and have already been used in the literature (see [7]).

Theorem 4.1. Under assumptions (U1)-(U7), we have

$$
\sup _{x \in \mathcal{S}_{\mathcal{F}}}\left|\widehat{m}_{\varphi}(x)-m_{\varphi}(x)\right|=O\left(h^{b}\right)+O_{a . s}\left(\sqrt{\frac{\ln d_{n}}{n \Phi(h)}}\right) .
$$


The proof of Theorem 4.1 is based on the same decomposition (1) and on the following lemmas

Lemma 4. Under the assumptions (U1)-(Ur), we get

$$
\sup _{x \in \mathcal{S}_{\mathcal{F}}}\left|r_{l}(x)-\tilde{m}_{l}(x)\right|=O_{a . s .}\left(\sqrt{\frac{\ln d_{n}}{n \Phi(h)}}\right) .
$$

Proof. By following the same steps as the proof of Lemma 1 and using Lemma 2.2 in [7] we get our result.

Lemma 5. Under the assumptions (U1), (U2) and(U4), we obtain that

$$
\sup _{x \in \mathcal{S}_{\mathcal{F}}}\left|\boldsymbol{E}\left(\tilde{m}_{1}(x)\right)-m_{\varphi}(x)\right|=O\left(h^{b}\right) .
$$

Proof. Poof of Lemma 5 is similar to that of Lemma 2.

Lemma 6. i) Under the assumptions (U1)-(U7), we have

$$
\sup _{x \in \mathcal{S}_{\mathcal{F}}}\left|\tilde{m}_{1}(x)-\boldsymbol{E}\left(\tilde{m}_{1}(x)\right)\right|=O_{a . c o}\left(\sqrt{\frac{\ln d_{n}}{n \Phi(h)}}\right) .
$$

ii) If assumptions (U1), (U3)-(U6) are satisfied, we get

$$
\begin{gathered}
\sup _{x \in \mathcal{S}_{\mathcal{F}}}\left|\tilde{m}_{0}(x)-1\right|=O_{\text {a.co }}\left(\sqrt{\frac{\ln d_{n}}{n \Phi(h)}}\right) \quad \text { and } \\
\exists \vartheta>0, \quad \text { such that } \sum_{n=1}^{\infty} \boldsymbol{P}\left(\inf _{x \in \mathcal{S}_{\mathcal{F}}} \tilde{m}_{0}(x)<\vartheta\right)<\infty .
\end{gathered}
$$

Proof. By considering the same decompositions and notations (2)-(5), following the same steps as in the proof of Lemma 3 and using Lemma 6 (i) in [7] instead of Lemma A.1 (i) in [1], we get under assumptions (U1)-(U4) and (U6)

$$
\sup _{x \in \mathcal{S}_{\mathcal{F}}} Q(x)=O(1) \quad \text { and } \sup _{x \in \mathcal{S}_{\mathcal{F}}} \mathbf{E}\left(M_{p, l}(x)\right)=O(1)
$$

uniformly on $x$, for $p=2,3,4$ and $l=0,1$,

$$
\sup _{x \in \mathcal{S}_{\mathcal{F}}}\left|\mathbf{E}\left(M_{2,1}(x)\right) \mathbf{E}\left(M_{4,0}(x)\right)-\mathbf{E}\left(M_{2,1}(x) M_{4,0}(x)\right)\right|=O\left(\frac{1}{n \Phi(h)}\right)
$$

and

$$
\sup _{x \in \mathcal{S}_{\mathcal{F}}}\left|\mathbf{E}\left(M_{3,1}(x)\right) \mathbf{E}\left(M_{3,0}(x)\right)-\mathbf{E}\left(M_{3,1}(x) M_{3,0}(x)\right)\right|=O\left(\frac{1}{n \Phi(h)}\right),
$$

which is, using hypothesis (U5), equals to $O\left(\sqrt{\frac{\ln d_{n}}{n \Phi(h)}}\right)$.

Now we prove that

$$
\sup _{x \in \mathcal{S}_{\mathcal{F}}}\left|M_{p, l}(x)-\mathbf{E}\left(M_{p, l}(x)\right)\right|=O_{a . c o}\left(\sqrt{\frac{\ln d_{n}}{n \Phi(h)}}\right) .
$$


To this end, we need the following decomposition.

Let be $j(x)=\arg \min _{j \in\left\{1,2, \ldots, d_{n}\right\}} d\left(x, x_{j}\right)$; we have

$$
\begin{aligned}
\sup _{x \in \mathcal{S}_{\mathcal{F}}}\left|M_{p, l}(x)-\mathbf{E}\left(M_{p, l}(x)\right)\right| \leqslant & \sup _{x \in \mathcal{S}_{\mathcal{F}}}\left|M_{p, l}(x)-M_{p, l}\left(x_{j(x)}\right)\right|+ \\
& +\sup _{x \in \mathcal{S}_{\mathcal{F}}}\left|M_{p, l}\left(x_{j(x)}\right)-\mathbf{E}\left(M_{p, l}\left(x_{j(x)}\right)\right)\right|+ \\
& +\sup _{x \in \mathcal{S}_{\mathcal{F}}}\left|\mathbf{E}\left(M_{p, l}\left(x_{j(x)}\right)\right)-\mathbf{E}\left(M_{p, l}(x)\right)\right| \\
& :=D_{1}^{p, l}+D_{2}^{p, l}+D_{3}^{p, l} .
\end{aligned}
$$

Using (U1), (U3) and (U4), we get

$$
D_{1}^{p, l} \leqslant \frac{C r_{n}}{n h \Phi(h)} \sup _{x \in \mathcal{S}_{\mathcal{F}}} \sum_{i=1}^{n}\left|\varphi^{l}\left(Y_{i}\right)\right| 1_{B(x, h) \cup B\left(x_{j(x)}, h\right)}\left(X_{i}\right) .
$$

Taking

$$
Z_{i}=\frac{C r_{n}}{h \Phi(h)}\left|\varphi^{l}\left(Y_{i}\right)\right| \sup _{x \in \mathcal{S}_{\mathcal{F}}} 1_{B(x, h) \cup B\left(x_{j(x)}, h\right)}\left(X_{i}\right) ;
$$

The assumption (U7) allows us to write

$$
\mathbf{E}\left|Z_{1}^{m}\right| \leqslant \frac{C r_{n}^{m}}{h^{m} \Phi^{m-1}(h)} .
$$

Using Corollary A.8 (ii) in [3] with $a_{n}^{2}=\frac{r_{n}}{h \Phi(h)}$, we get

$$
\frac{1}{n} \sum_{i=1}^{n} Z_{i}=\mathbf{E}\left(Z_{1}\right)+O_{a . c o}\left(\sqrt{\frac{r_{n} \ln n}{n h \Phi(h)}}\right) .
$$

Applying (6) again (for $\mathrm{m}=1$ ), one gets

$$
D_{1}^{p, l}=O\left(\frac{r_{n}}{h}\right)+O_{a . c o}\left(\sqrt{\frac{r_{n} \ln n}{n h \Phi(h)}}\right) .
$$

Combining this last result with assumption (U5) and the second part of the assumption (U1), we obtain

$$
D_{1}^{p, l}=O_{a . c o}\left(\sqrt{\frac{\ln d_{n}}{n \Phi(h)}}\right) .
$$

For the term $D_{3}^{p, l}$, since

$$
D_{3}^{p, l} \leqslant \mathbf{E}\left(\sup _{x \in \mathcal{S}_{\mathcal{F}}}\left|M_{p, l}(x)-M_{p, l}\left(x_{j(x)}\right)\right|\right)
$$

thus

$$
D_{3}^{p, l}=O_{a . c o}\left(\sqrt{\frac{\ln d_{n}}{n \Phi(h)}}\right) .
$$

And finally for the term $D_{2}^{p, l}$, we have For all $\eta>0$

$$
\begin{aligned}
\mathbf{P}\left(D_{2}^{p, l}>\eta \sqrt{\frac{\ln d_{n}}{n \Phi(h)}}\right) & =\mathbf{P}\left(\max _{j \in\left\{1, \ldots, d_{n}\right\}}\left|M_{p, l}\left(x_{j(x)}\right)-\mathbf{E}\left(M_{p, l}\left(x_{j(x)}\right)\right)\right|>\eta \sqrt{\frac{\ln d_{n}}{n \Phi(h)}}\right) \leqslant \\
\leqslant & d_{n} \times \max _{j \in\left\{1, \ldots, d_{n}\right\}} \mathbf{P}\left(\left|M_{p, l}\left(x_{j(x)}\right)-\mathbf{E}\left(M_{p, l}\left(x_{j(x)}\right)\right)\right|>\eta \sqrt{\frac{\ln d_{n}}{n \Phi(h)}}\right) . \\
- & 489-
\end{aligned}
$$


Taking for $p=2,3,4$

$$
\Upsilon_{p, i}=\frac{1}{h^{p-2} \Phi_{x}(h)}\left[\frac{\mu K_{i}\left(x_{j(x)}\right) \beta_{i}^{p-2}\left(x_{j(x)}\right) \varphi^{l}\left(Y_{i}\right)}{G\left(Y_{i}\right)}-\mathbf{E}\left(\frac{\mu K_{i}\left(x_{j(x)}\right) \beta_{i}^{p-2}\left(x_{j(x)}\right) \varphi^{l}\left(Y_{i}\right)}{G\left(Y_{i}\right)}\right)\right] .
$$

Using the binomial Theorem and hypothesis (U1), (U2) and (U7), we obtain for $p=2,3,4$

$$
\mathbf{E}\left|\Upsilon_{p, i}\right|^{m}=O\left(\Phi^{-m+1}(h)\right)
$$

So, we can apply a Bernstein-type inequality as done in the Corollary A.8 (i) in [3], to obtain

$$
\mathbf{P}\left(\frac{1}{n}\left|\sum_{i=1}^{n} \Upsilon_{p, i}\right|>\eta \sqrt{\frac{\ln d_{n}}{n \Phi(h)}}\right) \leqslant 2 \exp \left(-C \eta^{2} \ln d_{n}\right) .
$$

Thus, by choosing $\beta$ such that $C \eta^{2}=\beta$, we get

$$
\mathbf{P}\left(D_{2}^{p, l}>\eta \sqrt{\frac{\ln d_{n}}{n \Phi(h)}}\right) \leqslant C d_{n}^{1-\beta} .
$$

Then, hypothesis (U5) allows us to write

$$
D_{2}^{p, l}=O_{a . c o}\left(\sqrt{\frac{\ln d_{n}}{n \Phi(h)}}\right) .
$$

Finally, the result of lemma (6) follows from the relations (7), (8) and (9).

The second part of the lemma (6) can be directly deduced from the proof of the first one such that $\mathbf{E}\left(\tilde{m}_{0}(x)\right)=1$. For the last part, it comes straightforward that

$$
\begin{gathered}
\inf _{x \in \mathcal{S}_{\mathcal{F}}} \tilde{m}_{0}(x)<\frac{1}{2} \Rightarrow \exists x \in \mathcal{S}_{\mathcal{F}} \text { such that } \\
1-\tilde{m}_{0}(x)>\frac{1}{2} \Rightarrow \sup _{x \in \mathcal{S}_{\mathcal{F}}}\left|1-\tilde{m}_{0}(x)\right|>\frac{1}{2} \Rightarrow \sum_{n=1}^{\infty} \mathbf{P}\left(\inf _{x \in \mathcal{S}_{\mathcal{F}}} \tilde{m}_{0}(x)<\frac{1}{2}\right)<\infty .
\end{gathered}
$$

Theorem 4.1 is proved.

The authors would like to thank the Editor and the anonymous reviewer for their valuable comments.

\section{References}

[1] J.Barrientos-Marin, F.Ferraty, P.Vieu, Journal of Nonparametric Statistics, 22(2010), no. 5, 617-632. DOI: $10.1080 / 10485250903089930$

[2] S.Derrar, A.Laksaci, E.Ould Saïd, Journal of Statistical Theory and Practice, 9(2015), no. 4, 823-849. DOI: $10.1080 / 15598608.2015 .1032455$

[3] F.Ferraty, P.Vieu, Nonparametric functional data analysis: theory and practice, Springer Science \& Business Media, 2006.

[4] S.He, G.L.Yang, Estimation of the truncation probability in the random truncation model, Annals of Statistics, 1998, 1011-1027. 
[5] N.Helal, E.Ould-Sä̈d, Kernel conditional quantile estimator under left truncation for functional regressors, Opuscula Mathematica, 36(2016), 25-48.

[6] M.Lemdani, E.Ould-Said, Asymptotic behavior of the hazard rate kernel estimator under truncated and censored data, Communications in Statistics - Theory and Methods, 36(2007), no. 1, 155-173.

[7] S.Leulmi, F.Messaci, Journal of Siberian Federal University. Mathematics abd Physics, 12(2019), 379-391. DOI: 10.17516/1997-1397-2019-12-3-379-391

[8] S.Leulmi, F.Messaci, Local linear estimation of a generalized regression function with functional dependent data Communications in Statistics - Theory and Methods, 47(2018), no. $23,5795-5811$.

[9] F.Messaci, N.Nemouchi, I.Ouassou, M.Rachdi, Statistical Methods \& Applications, 24(2015), no. 4, 597-622. DOI:10.1007/s10260-015-0296-9

[10] W.Stute, Almost sure representations of the product-limit estimator for truncated data, The Annals of Statistics, 21(1993), no. 1, 146-156.

[11] M.Woodroofe, Estimating a distribution function with truncated data, The Annals of Statistics, 13(1985), no. 1, 163-177.

[12] X.Xiong, P.Zhou, C.Ailian, Asymptotic normality of the local linear estimation of the conditional density for functional time-series data, Communications in Statistics - Theory and Methods, 47(2018), no. 14, 3418-3440.

\title{
Скорость почти надежной сходимости обобщенной регрессионной оценки на основе усеченных и функциональных данных
}

\author{
Халима Будада \\ Сара Леулми \\ Университет Фрер Мантури \\ Константине 1, Алжир \\ Соумиа Харфучи \\ Университет Салах Бубнидер \\ Константине 3, Алжир
}

\begin{abstract}
Аннотация. В этой статье предлагается непараметрическая оценка обобщенной функции регрессии. Случайная переменная реального ответа (r.v.) подвергается усечению влево другим r.v., в то время как ковариата принимает свои значения в бесконечномерном пространстве. При стандартных предположениях устанавливаются точечные и равномерные почти наверняка сходимости предлагаемой оценки.
\end{abstract}

Ключевые слова: функциональные данные, усеченные данные, почти уверенная сходимость, локальная линейная оценка. 